\title{
Reconstruction of an optical inhomogeneity map improves fluorescence diffuse optical tomography
}

\author{
Nicolas Ducros ${ }^{1}$, Teresa Correia ${ }^{2}$, Andrea Bassi ${ }^{3}$, Gianluca \\ Valentini $^{3}$, Simon Arridge ${ }^{2}$, Cosimo D'Andrea ${ }^{3,4}$ \\ ${ }^{1}$ Univ Lyon, INSA-Lyon, Université Claude Bernard Lyon 1, UJM-Saint Etienne, \\ CNRS, Inserm, CREATIS UMR 5220, U1206, F-69621, LYON, France \\ ${ }^{2}$ Centre for Medical Image Computing, University College London, Malet Place, \\ London WC1E 6BT, United Kingdom \\ ${ }^{3}$ Istituto di Fotonica e Nanotecnologie (IFN-CNR) - Dipartimento di Fisica, \\ Politecnico di Milano, Piazza Leonardo da Vinci 32, I-20133 Milan, Italy \\ ${ }^{4}$ Center for Nano Science and Technology@PoliMi, Istituto Italiano di Tecnologia, \\ Via Giovanni Pascoli, 70/3, 20133 Milano \\ E-mail: nicolas.ducros@insa-lyon.fr
}

Submitted in March 2016

\begin{abstract}
We propose a new reconstruction algorithm for fluorescence diffuse optical tomography, which is designed for highly heterogeneous objects, such as biological tissues. It is a two-step algorithm that exploits continuous-wave measurements acquired at both excitation and fluorescence wavelengths. First, an optical inhomogeneity map, which depends on both absorption and diffusion coefficients, is obtained from excitation measurements. Second, the fluorescence distribution is reconstructed considering the recovered optical inhomogeneity map. The algorithm includes dimensionality reduction techniques, namely measurement compression and structured illumination, which significantly reduce acquisition and reconstruction times. The algorithm has been tested on experimental data acquired from tissuemimicking phantoms considering sparsity priors. We demonstrate the feasibility and effectiveness of this new approach that allows the fluorescence reconstruction quality to be improved with respect to that provided by the standard normalized Born method.
\end{abstract}

Keywords: fluorescence imaging, optical tomography, image reconstruction, heterogeneous objects

\section{Introduction}

Fluorescence diffuse optical tomography (FDOT), also known as fluorescence molecular tomography (FMT), is an optical technique that allows the 3D reconstruction of the distribution of exogenous fluorescent markers. It has been mainly adopted for in vivo preclinical imaging in small animals [1-3]. FDOT consists in illuminating a sample with 
light patterns (e.g. points or more complex shapes) and detecting the fluorescence light exiting the sample [4;5]. Multiple illumination-detection combinations are generally considered for different angles of view of the sample, which improves the spatial resolution of the reconstruction[6-8].

As discussed in [9], tomographic reconstruction of the fluorescent marker distribution generally consists in i) solving the forward problem that describes light propagation through the sample and ii) solving the inverse problem given a forward model. The inverse problem is very ill-posed and thus fluorescence reconstruction highly depends on the accuracy of the forward model itself [10].

The diffusion approximation is generally adopted to solve the forward problem. This partial differential equation, which may be solved by means of the finite element method $[11 ; 12]$ or the boundary element method [13; 14], requires the knowledge of several optical parameters, principally the absorption coefficient $\mu_{\mathrm{a}}$ and reduced scattering coefficient $\mu_{\mathrm{s}}^{\prime}$ at each optical wavelength being measured, as well as the refractive index of the medium, insofar as it affects the determination of correct boundary conditions. One of the major difficulties of FDOT is to estimate the distribution of these optical parameters, which is even more critical when highly heterogeneous objects such as biological samples (e.g. mouse) are considered.

One practical solution has been to assume the sample to be optically homogeneous and consider the ratio between measurements at fluorescence and excitation wavelength. The so-called normalized Born (nBorn) method was originally proposed by Ntziachritos et. al. [15] to correct for experimental factors such as detector gains and coupling losses. Interestingly, it also proved efficient to minimize the fluorescence reconstruction artifacts that appear in the presence of optical heterogeneities [16]. Although the state-of-the-art nBorn approach is effective in the case of absorbing heterogeneities, it presents severe limitations when scattering heterogeneities are present $[17 ; 18]$.

In the last years, FDOT research has focused on multi-modality approaches such as X-ray computed tomography-FDOT (CT-FDOT) [19-21], X-ray phase-contrast CTFDOT [22], magnetic resonance imaging-FDOT (MRI) [23; 24], single photon emission computed tomography-CT-FDOT [25], or positron emission tomography-FDOT [26]. Traditionally, the volume obtained from the concurrent modality (e.g. CT or MRI) is segmented into different anatomical segments (e.g. organs or tissue types) that can be used to constrain the resolution of the FDOT problem [19-22; 27; 28]. Semi-automatic approaches eliminating the need for prior image segmentation have also been proposed [29]. More recently, the knowledge of the anatomical segments has been exploited to improve the accuracy of the forward model, which results in an improved reconstruction quality. This is usually done by a segment-and-assign strategy, which consists in assigning a couple of optical properties to each of the anatomical segments prior to FDOT reconstruction. In [22], segmentation is performed from a phase-contrast CT scan of the mouse ex-vivo, while conventional CT [25; 30] or MRI [23; 31] acquired in vivo can be also considered. Segmentation is usually limited to four to six segments. However, choosing the right optical parameters for each segment is very challenging. 
Despite many studies, the optical properties of the tissues have not yet been fully established, due to inter-subject variability and measurements being predominantly performed using ex vivo tissues. Moreover, anatomical information provided by a non-optical concurrent imaging modality is only partially correlated with the optical parameters.

Therefore, it is highly desirable to recover the optical properties of the heterogeneities from optical measurements, which can be done by performing a diffuse optical tomography (DOT) reconstruction at the excitation wavelength [32-38]. However, continuous wave $(\mathrm{CW})$ measurements at a single wavelength cannot resolve both absorption and scattering coefficients [39]. Although it is possible to reconstruct only the absorption coefficient while assigning prior values to the scattering coefficient [38], effective unmixing of absorption and scattering requires time-resolved/frequency domain or multispectral measurements. Unfortunately, these two approaches show various drawbacks, such as the complexity of the experimental set-up, a lower signalto-noise ratio, and huge data sets that are difficult to be managed by the inversion algorithms. In practice, FDOT measurements are often performed in CW mode, especially in the case of a multi-modality instrumentation for which technical constraints push towards the use of simple experimental systems

The authors recently proposed the idea to reconstruct the so-called optical inhomogeneity map from CW DOT measurements and then to solve the FDOT problem [40]. The inhomogeneity map, which depends on both the absorption and scatting of the optical heterogeneities, allows the inaccuracies in the homogeneous FDOT forward problem to be corrected. Capitalizing on this work, we propose a new reconstruction method for FDOT, namely the inhomogeneous nBorn (i-nBorn) method, that generalizes the nBorn method. This new method is based on the joint solution of two inverse problems, which are both ill-posed and require the use of regularization schemes to recover an acceptable solution.

In this contribution, we describe a two-step reconstruction algorithm for the inBorn method. The first step implements a projected Gauss-Newton algorithm, which can accommodate different types of penalty terms, to reconstruct the sample inhomogeneities. The second step, which includes the inhomogeneity map recovered at the first step in the forward model, consists in the fluorescence image reconstruction using anisotropic diffusion (AD) regularization. To the best of our knowledge, this is the first implementation of the i-nBorn method to be fed up with experimental data.

The algorithm we propose easily integrates the use of structured light illumination and measurement compression, which are dimension reduction approaches that greatly reduce the acquisition and reconstruction times. Structured light is based on the illumination of the object by a small number of light patterns. Patterns can be chosen among natural basis such as Fourier [41] or wavelet [42], using singular value decomposition [43; 44] or applying the compressed sensing paradigm [45]. Different reconstructions schemes have been designed to handle large data sets, e.g. analytic method [46] or matrix free methods [28; 47]. Another approach is measurement 
compression, which can be considered as a preprocessing step where the measured images are (wavelet) compressed and the most significant basis function are retained to build the forward model with reduced dimensionality [5; 48].

The paper is organized as follows. In Section 2, we introduce the i-nBorn approach that is designed for fluorescence reconstruction in the presence of optical heterogeneities. In Section 3, we present an overview of our reconstruction method, which is based on the resolution of two inverse problems. First, we introduce dimensionality reduction through measurement compression. Then, we formalize the two inverse problems and explain how we solve them. Next, we describe how to build the forward problems. Finally, we provide a detailed description of our algorithm. In Section 4, we discuss the phantom experiments and the figures of merit of the reconstructions. We present our results in Section 5, discuss them in Section 6, and conclude in Section 7.

\section{Fluorescence diffuse optical tomography in the presence of heterogeneities}

We consider an object domain $\Omega$ with absorption coefficient $\mu_{\mathrm{a}}(\mathbf{r}), \mathbf{r} \in \Omega$, and reduced scattering coefficient $\mu_{\mathrm{s}}^{\prime}(\mathbf{r}), \mathbf{r} \in \Omega$, that has embedded within it a fluorescent dye. Let $h(\mathbf{r}), \mathbf{r} \in \Omega$, be the unknown fluorescence yield, which is related to the concentration of the fluorescent dye. The FDOT image reconstruction problem consists in solving the following inverse problem

$$
\Gamma^{\mathrm{f}}=A^{\mathrm{f}} h,
$$

where $\Gamma^{\mathrm{f}}$ is the fluorescence signal measured at the emission wavelength $\lambda^{\mathrm{f}}$ and $A^{\mathrm{f}}$ is the forward operator.

\section{1. nBorn method}

The forward operator $A^{\mathrm{f}}$ strongly depends on the optical properties of the object, which are unknown. If the operator $A^{\mathrm{f}}$ is built from estimated optical properties, the resulting model error dramatically degrades the reconstruction quality. A practical way of alleviating this problem has been to normalize data, which leads to the normalized FDOT problem

$$
\Gamma^{\mathrm{n}}=A^{\mathrm{n}} h, \quad \text { with } \Gamma^{\mathrm{n}}=\frac{\Gamma^{\mathrm{f}}}{\Gamma^{\mathrm{x}}},
$$

where $\Gamma^{\mathrm{x}}$ is the signal measured at the excitation wavelength $\lambda^{\mathrm{x}}$ and $A^{\mathrm{n}}$ is the normalized forward operator computed assuming homogeneous optical properties. Despite the very good results obtained in many configurations, it has been shown that the nBorn method does not compensate for the effect of unknown optical heterogeneities, especially scattering $[17 ; 18]$. 


\subsection{Full DOT-FDOT approach}

One possibility to improve the nBorn method is to address the full DOT-FDOT problem for which $\mu_{\mathrm{a}}, \mu_{\mathrm{s}}^{\prime}$ and $h$ are all recovered and refractive index is assumed known [32-36]. It consists in jointly (either sequentially or simultaneously) solving a pair of inverse problems. When the optical properties of the object are assumed to be the same at $\lambda^{\mathrm{x}}$ and $\lambda^{\mathrm{f}}$ we have

$$
\begin{aligned}
& \Gamma^{\mathrm{x}}=F\left(\mu_{\mathrm{a}}, \mu_{\mathrm{s}}^{\prime}\right) \quad \text { and } \\
& \Gamma^{\mathrm{n}}=A^{\mathrm{n}}\left(\mu_{\mathrm{a}}, \mu_{\mathrm{s}}^{\prime}\right) h .
\end{aligned}
$$

where $F$ is the (non-linear) excitation forward model. The pair of maps $\left(\mu_{\mathrm{a}}, \mu_{\mathrm{s}}^{\prime}\right)$ may be recovered from the DOT problem of $(3 \mathrm{a})$ and then used to build $A^{\mathrm{n}}$ in the FDOT problem of $(3 \mathrm{~b})$. However, when $\mathrm{CW}$ measurements are considered, the DOT problem has no unique solution [39]. Different pairs of maps $\left(\mu_{\mathrm{a}}, \mu_{\mathrm{s}}^{\prime}\right)$ may satisfy (3a) while resulting into a fluorescence forward model $A^{\mathrm{n}}$ that is subject to model error.

\subsection{Inhomogeneous nBorn approach}

In a previous work [40], we suggested an approach that allows DOT-FDOT reconstruction to be performed from $\mathrm{CW}$ data. The key idea is to recast the forward problem from a solution to a diffusion equation into a solution of the equivalent Helmholtz equation

$$
\left(\nabla^{2}+\eta\right) \Psi=s
$$

where $\eta$ is a scalar optical property with units of reciprocal squared length, defined as $\eta=\nabla^{2}\left(\kappa^{\frac{1}{2}}\right) / \kappa^{\frac{1}{2}}+\mu_{\mathrm{a}} / \kappa, \kappa=\frac{1}{3}\left(\mu_{\mathrm{s}}^{\prime}+\mu_{\mathrm{a}}\right)^{-1}$ is the diffusion coefficient inside $\Omega$ and $\Psi$ and $s$ are the transformed fluence and source terms. The system of equations (3a) $-(3 \mathrm{~b})$ may now be rewritten as

$$
\begin{aligned}
& \Gamma^{\mathrm{x}}=G(\eta), \\
& \Gamma^{\mathrm{n}}=B(\eta) \Upsilon,
\end{aligned}
$$

where $\Upsilon$ is a modified fluorescence yield that is given by

$$
\Upsilon=\frac{h}{\kappa} .
$$

The strategy to recover $h$ is the following. First, the nonlinear problem of $(5 \mathrm{a})$ is solved and an optical inhomogeneity map $\eta$, which depends on the optical heterogeneities of the medium, is obtained. Second, the $\eta$ map is used to build the forward model $B$ and the linear problem of $(5 b)$ is solved, providing the $\Upsilon$ map. Finally, the unknown fluorescence yield is given by

$$
h=\hat{\kappa} \Upsilon
$$


where $\hat{\kappa}$ is chosen, in practice, as an estimate for $\kappa$. In this manuscript, we assume $\kappa$ is slowly varying. Hence, we have the following approximation:

$$
\hat{\kappa} \simeq \frac{\hat{\mu}_{\mathrm{a}}}{\eta}
$$

where $\hat{\mu}_{\mathrm{a}}$ is an estimate for the absorption coefficient, chosen as a constant.

\section{Reconstruction overview}

\subsection{Data Acquisition and Compression}

The object $\Omega$ is illuminated at $I$ view angles. At each angle, a set of $J$ source patterns is projected onto the object. Such an acquisition results in a set of $I J$ excitation images and $I J$ fluorescence images. Let $\mathbf{m}_{i, j}^{\mathrm{x}}$ and $\mathbf{m}_{i, j}^{\mathrm{f}}$ be the excitation and fluorescence images, respectively, measured at the $i$ th view after illumination with the $j$ th source pattern. In the following sections, we use a vector representation for the images, i.e. $\mathbf{m}_{i, j}^{\mathrm{x}} \in \mathbb{R}^{P}$

and $\mathbf{m}_{i, j}^{\mathrm{f}} \in \mathbb{R}^{P}$, where $P$ is the number of pixels. The normalized fluorescence image is defined by

$$
\mathbf{m}_{i, j}^{\mathrm{n}}=\frac{\mathbf{m}_{i, j}^{\mathrm{f}}}{\mathbf{m}_{i, j}^{\mathrm{x}}},
$$

where the division is component-wise (pixel-wise). Each of the $I J$ excitation images, as well as each of the $I J$ normalized fluorescence images, are compressed by applying a wavelet transform $\mathbf{D}$ and retaining their $K$ most relevant wavelet components. The data for each forward problem then consists of the $I J K$ retained components stacked into a vector with components

$$
\Gamma_{i, j, k}=\left\langle\mathbf{d}_{i, j, k}, \mathbf{m}_{i, j}\right\rangle
$$

where $\mathbf{d}_{i, j, k}^{\mathrm{f}} \in \mathbb{R}^{P}$ is the wavelet vector associated with the $k^{\text {th }}$ component of the image acquired from the $j^{\text {th }}$ illumination pattern at the $i^{\text {th }}$ angle.

\subsection{Inverse problems}

We consider the reconstruction of the unknown quantities $\boldsymbol{\eta} \in \mathbb{R}^{N}$ and $\mathbf{h} \in \mathbb{R}^{N}$, where $N$ is the number of voxels, from the measured data $\Gamma^{\mathrm{x}} \in \mathbb{R}^{I J K}$ and $\Gamma^{\mathrm{n}} \in \mathbb{R}^{I J K}$ given the discrete forward models $\mathcal{G}$ and $\mathbf{B}$. The unknown quantities are obtained minimizing the cost functions

$$
\begin{aligned}
\mathcal{L}_{\text {dot }}(\boldsymbol{\eta}) & =\frac{1}{2}\left\|\boldsymbol{\Gamma}^{\mathbf{x}}-\mathcal{G}(\boldsymbol{\eta})\right\|^{2}+\alpha \mathcal{R}_{\mathrm{dot}}(\boldsymbol{\eta}) \quad \text { and } \\
\mathcal{L}_{\text {fdot }}(\boldsymbol{\Upsilon}) & =\frac{1}{2}\left\|\boldsymbol{\Gamma}^{\mathrm{n}}-\mathbf{B} \boldsymbol{\Upsilon}\right\|^{2}+\beta \mathcal{R}_{\text {fdot }}(\boldsymbol{\Upsilon}),
\end{aligned}
$$

where $\alpha$ (resp. $\beta$ ) is a regularization parameter that sets the trade-off between the data fidelity $\frac{1}{2}\left\|\boldsymbol{\Gamma}^{\mathrm{x}}-\mathcal{G}(\boldsymbol{\eta})\right\|^{2}\left(\right.$ resp. $\left.\frac{1}{2}\left\|\boldsymbol{\Gamma}^{\mathrm{n}}-\mathbf{B} \boldsymbol{\Upsilon}\right\|^{2}\right)$ and the penalty function $\mathcal{R}_{\text {dot }}$ (resp. $\mathcal{R}_{\text {fdot }}$ ). 
Penalty functions have long been confined to $\ell_{2}$-norm regularization that promotes the smoothness of the solution [19-22; 49]. More recently, penalty functions based on $\ell_{1}$-norm have received a lot of attention, since they promote sparse solutions $[26 ; 35 ; 36 ; 50 ; 51]$. The DOT problem has also been formulated as a joint sparse recovery problem with a $\ell_{0}$-norm penalty, which assumes that optical heterogeneities have a small support [52].

In the following we present our minimization framework for both $\mathcal{L}_{\text {dot }}$ and $\mathcal{L}_{\text {fdot }}$. Remind that $\boldsymbol{\eta}$ is required to build $\mathbf{B}$, hence, (11a) has to be solved before (11b).

3.2.1. Solution of the DOT problem The DOT cost function given at (11a) is minimized using a Gauss-Newton algorithm with an inequality constrain of the form $\eta_{\min } \leq \eta$. The Gauss-Newton method is a traditional tool for non linear minimization, which iteratively solves $\min _{\boldsymbol{\eta}} \mathcal{L}(\boldsymbol{\eta})$ starting with an initial guess $\boldsymbol{\eta}^{0}$ and building new estimates $\boldsymbol{\eta}^{\ell+1}=\boldsymbol{\eta}^{\ell}+\tau^{\ell} \Delta \boldsymbol{\eta}^{\ell}$, where $\Delta \boldsymbol{\eta}^{\ell}$ is the Gauss-Newton step and $\tau^{\ell}$ is the step length.

$$
\left(\mathbf{J}^{\ell \top} \mathbf{J}^{\ell}+\alpha \mathbf{C}^{\ell}\right) \Delta \boldsymbol{\eta}^{\ell}=-\mathbf{g}^{\ell},
$$

where $\mathbf{J}^{\ell}$ is the Jacobian matrix of $\mathcal{G}$ about $\boldsymbol{\eta}^{\ell}$ and $\mathbf{C}^{\ell}$ is the Hessian matrix of $\mathcal{R}_{\text {dot }}$ about $\boldsymbol{\eta}^{\ell}$. The vector $\mathbf{g}^{\ell}$, which denotes the gradient of $\mathcal{L}$ at $\boldsymbol{\eta}^{\ell}$, is given by

$$
\mathrm{g}^{\ell}=-\mathbf{J}^{\ell \top}\left(\boldsymbol{\Gamma}^{\mathrm{n}}-\mathcal{G}^{\ell}\right)+\alpha \nabla \mathcal{R}_{\mathrm{dot}}^{\ell},
$$

where $\mathcal{G}^{\ell}=\mathcal{G}\left(\boldsymbol{\eta}^{\ell}\right)$ is the forward estimate and $\nabla \mathcal{R}_{\text {dot }}^{\ell}$ is the gradient of $\mathcal{R}_{\text {dot }}$ at $\boldsymbol{\eta}^{\ell}$. Assuming the optical inhomogeneity map to be piecewise constant, we choose

$$
\mathcal{R}_{\operatorname{dot}}(\boldsymbol{\eta})=\mathrm{TV}(\boldsymbol{\eta}),
$$

where TV is the total variation semi-norm. To ensure the global convergence of the algorithm, a line search along the direction $\Delta \boldsymbol{\eta}^{\ell}$ is performed, the best step length being retained. The inequality constrain has been implemented considering the fixed-variable method described in [53].

In the following, we denote $\hat{\boldsymbol{\eta}}$ the solution obtained after convergence of the iterative process.

3.2.2. Solution of the FDOT problem The FDOT cost function (11b) is minimized considering the split operator method introduced in [54], considering a regularization penalty of the form

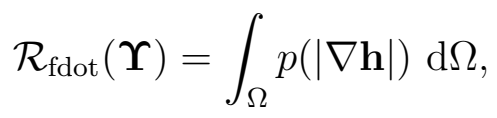

where $p$ is an edge-preserving potential function. The minimizer of (11b) is the solution of

$$
\mathbf{B}^{\top}\left(\mathbf{B} \Upsilon-\Gamma^{\mathrm{n}}\right)=-\beta \mathcal{D}(\Upsilon) \Upsilon
$$


where the right-hand side term is the nonlinear anisotropic diffusion with $\mathcal{D}(\mathbf{\Upsilon})=$ $-\nabla \cdot\left[\exp \left[-\left(\frac{|\nabla \Upsilon|}{T}\right)^{2}\right] \nabla\right]$, where the parameter $T$ is the threshold [54-56]. The solution is obtained using the two-step iterative method described in [54].

\subsection{Forward problems}

In this section, we discuss the calculation of the Jacobian matrix $\mathbf{J}^{\ell}$, forward estimate $\mathcal{G}^{\ell}$, and weight matrix B required in (12), (13), and (16), respectively. All the three quantities require an appropriate model for the propagation of light within the object $\Omega$.

Assuming $\kappa$ is known on the boundary $\partial \Omega$, we have the following discrete formulation for (4) using a finite element method (FEM) implementation [11]:

$$
\mathbf{H}(\boldsymbol{\eta}) \Psi=\mathbf{s}
$$

where $\mathbf{H}(\boldsymbol{\eta}) \in \mathbb{R}^{N \times N}$ is the Helmholtz operator that only depends on $\boldsymbol{\eta}, \boldsymbol{\Psi} \in \mathbb{R}^{N \times 1}$ is the pseudo photon density vector (which is related to the measured data as detailed in appendix $\mathrm{A}$ ), and $\mathbf{s} \in \mathbb{R}^{N \times 1}$ is the source vector. The propagation operator has been computed using the Matlab TOAST package [57].

The forward estimate $\mathcal{G}^{\ell}$ is of size $I J K \times 1$. The $(i, j, k)$ th entry of $\mathcal{G}^{\ell}$ is denoted $\mathcal{G}_{i, j, k}^{\ell} \in \mathbb{R}$. By definition, it can be computed as

$$
\mathcal{G}_{i, j, k}^{\ell}=\left\langle\mathbf{d}_{i, j, k}^{\mathrm{x}}, \boldsymbol{\psi}_{i, j}^{\ell}\right\rangle
$$

where $\boldsymbol{\psi}_{i, j}^{\ell}$ is obtained solving (17) with $\mathbf{s}=\mathbf{s}_{i, j}$ at iteration $\ell$.

The Jacobian matrix $\mathbf{J}^{\ell}$ is of size $I J K \times N$. The $(i, j, k)$ th row of $\mathbf{J}^{\ell}$ is denoted $\mathbf{j}_{i, j, k}^{\ell} \in \mathbb{R}^{N}$. It may be shown that $[9]$

$$
\mathbf{j}_{i, j, k}^{\ell}=\boldsymbol{\psi}_{i, j}^{\ell} \circ \boldsymbol{\psi}_{i, j, k}^{\ell},
$$

where $\boldsymbol{\psi}_{i, j, k}^{\ell}$ is the adjoint solution of (17) for $\mathbf{s}=\mathbf{d}_{i, j, k}^{\mathrm{x}}$ at iteration $\ell$. The symbol $\circ$ denotes the Hadamard product.

The weight matrix $\mathbf{B}$ is of size $I J K \times N$. The $(i, j, k)$ th row of $\mathbf{B}$ is denoted $\mathbf{b}_{i, j, k} \in \mathbb{R}^{N}$. As shown in appendix Appendix A, it can be computed as

$$
\mathbf{b}_{i, j, k}=\boldsymbol{\psi}_{i, j} \circ \boldsymbol{\psi}_{i, j, k}
$$

where $\boldsymbol{\psi}_{i, j}$ is the solution of (17) with $\mathbf{s}=\mathbf{s}_{i, j}$ at the final iteration of step 1 while $\boldsymbol{\psi}_{i, j, k}$ is the adjoint solution of (17) with $\mathbf{s}=\mathbf{d}_{i, j, k}^{\mathrm{n}} / \boldsymbol{\psi}_{i, j}$ (element-wise division).

\section{Material and methods}

\subsection{Experimental set-up}

In the following paragraph a brief description of the experimental set-up is reported. A more detailed description can be found in $[42 ; 58]$. The light emitted by a diode 

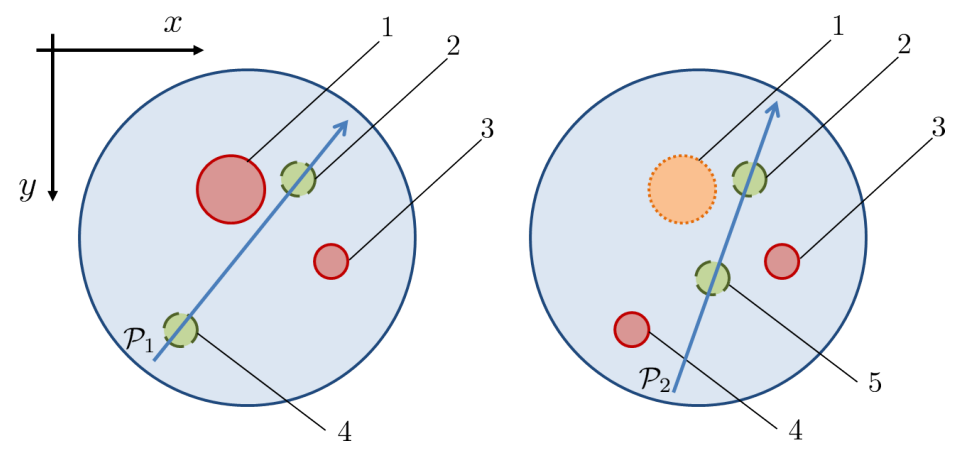

Figure 1. Bottom view of the epoxy resin phantoms used in the experiments. Phantom 1 is depicted on the left and phantom 2 on the right. Both phantoms are cylinders of diameter $20 \mathrm{~mm}$ and height $80 \mathrm{~mm}$. Red full line (-) indicates absorbing inclusions, green dashed line (--) fluorescence inclusions, and orange dotted line $(\cdots)$ scattering inclusions. The diameter and length of inclusions 1 to 5 are reported in Table 1 and 2. The paths $\mathcal{P}_{1}$ and $\mathcal{P}_{2}$ are used to plot profiles in Fig. 6.

\begin{tabular}{lrrrr}
\hline inclusion & 1 & 2 & 3 & 4 \\
\hline$\varnothing(\mathrm{mm})$ & 4 & 2 & 2 & 2 \\
$L(\mathrm{~mm})$ & 30 & 21 & 21 & 30 \\
\hline$\mu_{\mathrm{a}}\left(\mathrm{mm}^{-1}\right)$ & 0.20 & 0.02 & 0.20 & 0.02 \\
$\mu_{\mathrm{s}}^{\prime}\left(\mathrm{mm}^{-1}\right)$ & 1.00 & 1.00 & 1.00 & 1.00 \\
$\eta\left(\mathrm{mm}^{-2}\right)$ & 0.72 & 0.06 & 0.72 & 0.06 \\
dye & no & yes & no & yes \\
\hline
\end{tabular}

Table 1. Inclusions in phantom 1. The diameter $(\varnothing)$, length $(L)$, and optical properties of the heterogeneities are reported.

\begin{tabular}{lrrrrr}
\hline inclusion & 1 & 2 & 3 & 4 & 5 \\
\hline$\varnothing(\mathrm{mm})$ & 4 & 2 & 2 & 2 & 2 \\
$L(\mathrm{~mm})$ & 30 & 21 & 21 & 30 & 21 \\
\hline$\mu_{\mathrm{a}}\left(\mathrm{mm}^{-1}\right)$ & 0.02 & 0.02 & 0.20 & 0.20 & 0.02 \\
$\mu_{\mathrm{s}}^{\prime}\left(\mathrm{mm}^{-1}\right)$ & 5.00 & 1.00 & 1.00 & 1.00 & 1.00 \\
$\eta\left(\mathrm{mm}^{-2}\right)$ & 0.30 & 0.06 & 0.72 & 0.72 & 0.06 \\
dye & no & yes & no & no & yes \\
\hline
\end{tabular}

Table 2. Inclusions in phantom 2. The diameter $(\varnothing)$, length $(L)$, and optical properties of the heterogeneities are reported. 
laser (wavelength of $630 \mathrm{~nm}$, power $\approx 10 \mathrm{~mW}$ ) is spatially modulated by means of a digital micromirror device (DMD) (Discovery 1100 - ALP1, Vialux), which creates different patterns that are projected onto the sample surface through an objective lens. The sample is placed on a motorized rotational stage in order to acquire multiple views. In particular, measurements have been carried out on biological tissue mimicking phantoms. The light exiting the sample surface is collected by an objective lens (f $=50 \mathrm{~mm}, \mathrm{f} \#=2.8$, Nikon Co.) and projected on a low noise 16-bit cooled (-40 ${ }^{\circ} \mathrm{C}$ ) CCD camera (Versarray 512, Princeton Instruments). In order to discriminate fluorescence from excitation light, a high-pass filter (RG-695, Schott) in conjunction with an interference filter (XF 3076, Omega) have been inserted in front of the objective lens.

The phantom is an epoxy resin cylinder of diameter $20 \mathrm{~mm}$ and height $80 \mathrm{~mm}$. Appropriate concentrations of toner powder and $\mathrm{Ti}_{2}$ particles have been added to the resin so as to simulate the absorption and scattering parameters of biological tissues. The optical properties of the phantom have been measured by means of a time-resolved diffused optical spectroscopy system [59]. The following values have been obtained at $630 \mathrm{~nm}: \mu_{a}=0.02 \mathrm{~mm}^{-1}$ and $\mu_{s}^{\prime}=1 \mathrm{~mm}^{-1}$. In order to simulate inclusions, cylindrical holes have been drilled into the phantom at different positions (see Fig. 1). The diameter and length of the inclusions are reported in Table 1 and 2. Each of the inclusions can be independently filled with a liquid solution whose optical properties differ from the background. In the case of absorbing/scattering inclusions, the solution is made of ink and intralipid $\cap$ and by changing their concentrations it is possible to modify the absorption and scattering coefficients, respectively. Fluorescent inclusions have been obtained filling the holes with a fluorescent dye (Nile Blue dye concentration of $30 \mu \mathrm{M}$ ). In particular, two phantoms of increasing complexity have been prepared, as shown in Fig. 1. The optical properties of the inclusions for both phantoms are reported in Table 1 and 2. In the first phantom, two absorbing cylindrical inclusions (labels 1 and 3 according to Fig. 1) and two fluorescent inclusions (labels 2 and 4) have been inserted. In the second phantom, two absorbing inclusions (labels 3 and 4), one scattering inclusion (label 1), and two fluorescent inclusions (labels 2 and 5) have been inserted.

Measurements at both excitation and fluorescence wavelengths have been carried out in transmission every $18^{\circ}$, resulting in $I=20$ views. For simplicity, only $J=1$ uniform source pattern has been considered at each view angle.

\subsection{Practicalities}

Mesh The mesh used to solve the Helmholtz-like equation consists of 42886 tetrahedral elements connected by 7468 vertices and is displayed in Fig. 2a. It was obtained

considering the DMD-CCD-based method proposed in [42], which can handle any convex sample without requiring any extra device. The main steps are the following. First, uniform illumination patterns were projected onto the sample and the resulting shadow images were recorded rotating the sample with a step angle of $1^{\circ}$. Then, the obtained 
stack of shadow images was filtered backprojected, which results in a $3 \mathrm{D}$ volume that was thresholded to get a binary volume indicating the volume occupied by the sample. Finally, the iso2mesh mesh generator was employed [60] to compute the mesh from the binary volume.

Illumination/detection areas The illumination and detection areas (see Fig. $2 \mathrm{c}$ and d) are determined from the shadow images. For each measurement angle, the detection area is chosen as a rectangle that fits within the sample. To avoid camera saturation during excitation measurements, the detection area is narrower than the phantom diameter. The retained size was $34 \mathrm{~mm} \times 17 \mathrm{~mm}$. For each view, the illumination area is obtained by symmetry on the opposite side of the object (see Fig. 2b). Illumination and detection areas all start $2 \mathrm{~mm}$ away from the top of the phantom.

Image preprocessing The recorded excitation and fluorescence images are of size $256 \times 256$ (see Fig. $2 \mathrm{c}$ and 2 d). All of them are cropped to the detection area and resampled so as to get $128 \times 64$ rectangular images. Then, the fluorescence images are wavelet transformed (Daubechies 4 wavelet basis) using the wavelab software [61]. Finally, for each image, the $K=64$ most significant wavelet basis are retained to build the detection patterns $\mathbf{d}_{i, j, k}$ introduced in (10).

Reconstruction The grid we consider to reconstruct both the inhomogeneity and the fluorescence maps is regular and consists of $N=30 \times 29 \times 59$ voxels of size $(0.75 \mathrm{~mm})^{3}$. Note that this grid differ from the grid where the binary volume used to create the mesh is defined. We chose $\mu_{\mathrm{a}}=0.02 \mathrm{~mm}^{-1}$ and $\mu_{\mathrm{s}}^{\prime}=1 \mathrm{~mm}^{-1}$ to build the weight matrix of the nBorn method. The initial guess for $\eta$ required by the i-nBorn method was obtained considering the same values, i.e. choosing $\eta^{0}=3 \mu_{\mathrm{a}}\left(\mu_{\mathrm{a}}+\mu_{\mathrm{s}}^{\prime}\right) \approx 6 \times 10^{-2} \mathrm{~mm}^{-2}$. The estimate of the absorption coefficient $\hat{\mu}_{\mathrm{a}}$ was set to $0.02 \mathrm{~mm}^{-1}$ while the optimization bound $\eta_{\min }$ was set to $\frac{1}{2} \eta^{0}$.

\subsection{Performance metrics}

The reconstruction quality has been assessed by means of three main objective performance metrics. First, the Pearson correlation has been considered as a global metric that indicates how well two volumes are linearly correlated. Given the groundtruth $h^{\text {true }}$ and reconstructed fluorescence distribution $h^{\text {rec }}$, the Pearson correlation $\rho$ is defined by

$$
\rho=\frac{\operatorname{cov}\left(h^{\text {rec }}, h^{\text {true }}\right)}{\sigma\left(h^{\text {rec }}\right) \sigma\left(h^{\text {true }}\right)},
$$

where cov is the covariance and $\sigma$ the standard deviation. A large $\rho$ indicates a high correlation between the images.

Second, the contrast-to-noise ratio $(\mathrm{CNR})$ has been considered. It is defined by

$$
\mathcal{C}_{\text {roi,back }}=\frac{\mu_{\text {roi }}-\mu_{\text {back }}}{\left(w_{\text {roi }} \sigma_{\text {roi }}^{2}+w_{\text {back }} \sigma_{\text {back }}^{2}\right)^{1 / 2}},
$$




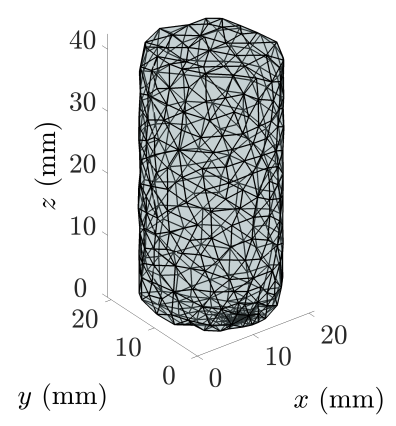

(a)

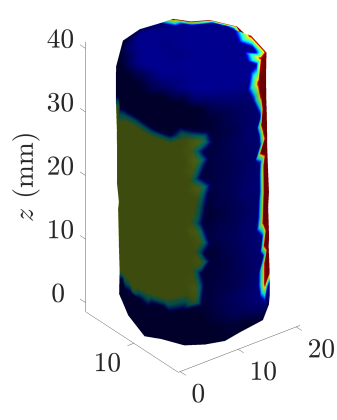

(b)

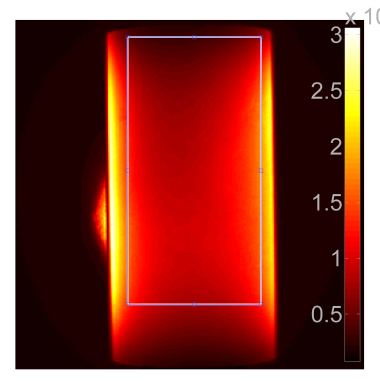

(c)

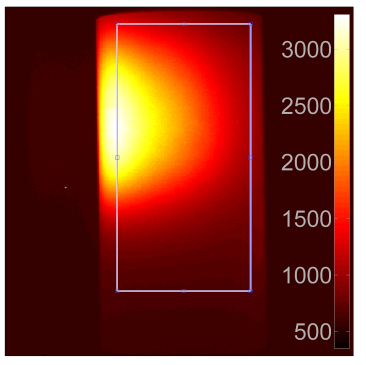

(d)

Figure 2. a) Mesh of the object b) Detection and illumination areas for the first view angle c) image at excitation wavelength d) image at fluorescence wavelength. The rectangles in (c) and (d) indicate the detection area.

where $\mu_{\text {roi }}\left(\mu_{\text {back }}\right)$ and $\sigma_{\text {roi }}\left(\sigma_{\text {back }}\right)$ are the average and standard deviation of $h^{\text {rec }}$ in the region-of-interest $\Omega_{\text {roi }}$ (background $\left.\Omega_{\text {back }}\right)$ and $w_{\text {fluo }}\left(w_{\text {back }}\right)$ is the ratio of the volume occupied by the two fluorescent inclusions (the rest of the volume).

We define the global $\mathrm{CNR} \mathcal{C}$ by choosing

$$
\Omega_{\mathrm{roi}}=\Omega_{\mathrm{A}} \cup \Omega_{\mathrm{B}} \quad \text { and } \quad \Omega_{\mathrm{back}}=\Omega \backslash \Omega_{\mathrm{roi}},
$$

where $\Omega_{A}$ (resp. $\Omega_{B}$ ) is the support of the the first (resp. second) fluorescent inclusion. The larger the CNR, the better.

Last, the relative quantification factor $\mathcal{Q}$ is introduced as a measure of the quantitativeness of the reconstruction. We define

$$
\mathcal{Q}=\min \left(\frac{\mathcal{C}_{\mathrm{A}}}{\mathcal{C}_{\mathrm{B}}}, \frac{\mathcal{C}_{\mathrm{B}}}{\mathcal{C}_{\mathrm{A}}}\right)
$$

where $\mathcal{C}_{\mathrm{A}}$ (resp. $\mathcal{C}_{\mathrm{B}}$ ) is the CNR of inclusion $\mathrm{A}$ (resp. B), which is obtained choosing $\Omega_{\text {roi }}=\Omega_{\mathrm{A}}$ (resp. $\left.\Omega_{\text {roi }}=\Omega_{\mathrm{B}}\right)$ and $\Omega_{\text {back }}=\Omega \backslash\left(\Omega_{\mathrm{A}} \cup \Omega_{\mathrm{B}}\right)$. The closer the relative quantification is to 1 , the better.

\subsection{Choice of the regularization parameters}

The reconstruction quality dramatically depends on the choice of the regularization parameters $\alpha$ and $\beta$ given in (11a) and (11b), respectively. While the best $\alpha$ has been chosen from visual inspection, $\beta$ has been chosen by optimizing an objective performance metric, which enables fair comparisons of the reconstructions. The retained performance metric is the global CNR.

\section{Results}

The fluorescence distribution $h$ was reconstructed from the same data sets considering both the i-nBorn method and the state-of-the-art nBorn method. The nBorn method 


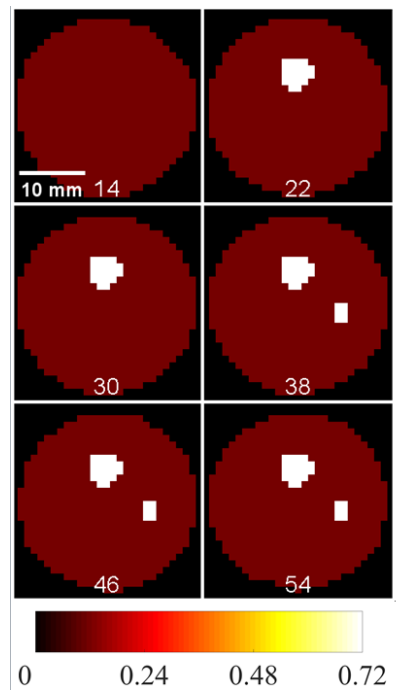

(a)

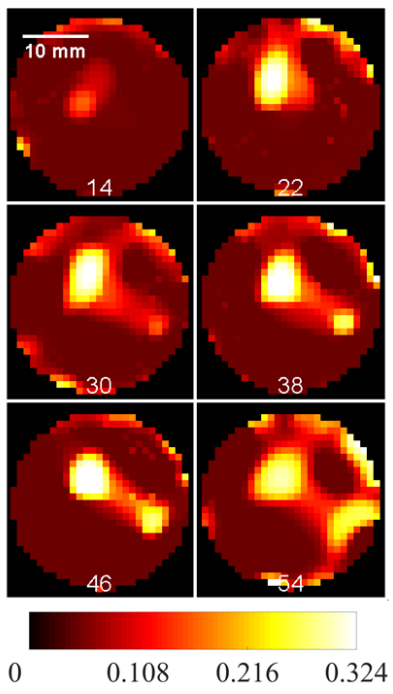

(b)

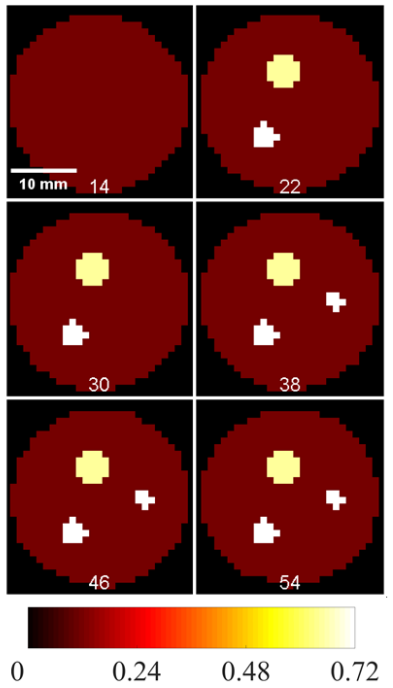

(c)

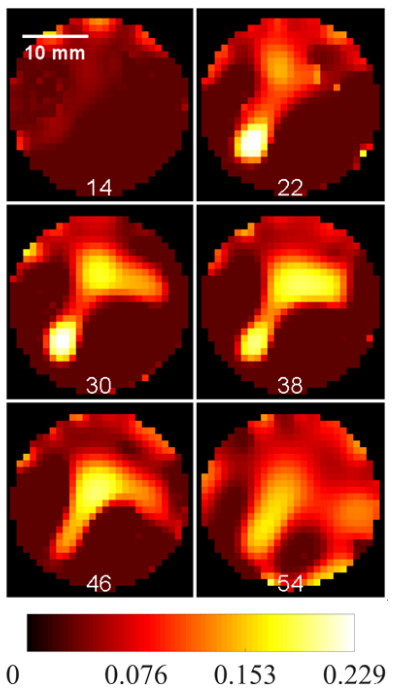

(d)

Figure 3. Optical inhomogeneity maps (in $\mathrm{mm}^{-2}$ ). a) ground truth in phantom 1 , b) reconstruction in phantom 1, c) ground truth in phantom 2, b) reconstruction in phantom 2. Slices at increasing $z$ are displayed from top left to bottom right. Sparcity priors (14) and (15) were considered.

requires to solve (2), which is an ill-posed inverse problem that requires regularization. To ensure fair comparison between the nBorn and the proposed i-nBorn method, we solve (2) and (5b) the same way, i.e., minimizing a cost function of the form (11b). To show the universality of our approach, not only the sparsity priors promoted by the regularizers given by (14) and (15) were considered, but also more classical regularity priors of the form $\mathcal{R}_{\text {dot }}(\boldsymbol{\eta})=\|\Delta \boldsymbol{\eta}\|_{2}^{2}$ and $\mathcal{R}_{\text {fdot }}(\boldsymbol{\Upsilon})=\|\Upsilon\|_{2}^{2}[19-22 ; 49]$.

Slices of the inhomogeneity maps reconstructed in both phantoms are shown in Fig. 3. Three-dimensional rendering (Volume Viewer ImageJ plugin) of the fluorescence reconstructions in phantom 1 and phantom 2 are reported in Fig. 4 and 5, respectively. For both phantom reconstructions, the fluorescence profiles obtained along paths $\mathcal{P}_{1}$ and $\mathcal{P}_{2}$ (refer to Fig. 1 to localize the paths) are depicted in Fig. 6 considering sparsity priors as well as regularity priors. Finally, the performance metrics introduced in section 4.3 were used to evaluate reconstructions in both phantoms considering the two approaches. The metrics are reported in Table 3 for reconstructions performed with sparsity priors and in Table 4 for reconstructions performed with regularity priors.

In phantom 1, we observe a clear advantage of the proposed approach over the standard nBorn method as can be observed visually in the 3D rendering of Fig. 4 and in the profiles of the top row of Fig. 6. In particular, the nBorn reconstruction is unable to resolve separately the two inclusions as our proposed approach does. This is quantitatively confirmed with the increase of the global CNR of the reconstructed volume $\mathcal{C}$ and its correlation with the ground truth $\rho$ (see Table 3 and Table 4). Specifically, we observe a $36 \%$ increase (resp. $23 \%$ ) of $\rho$ and a $42 \%$ increase (resp. 

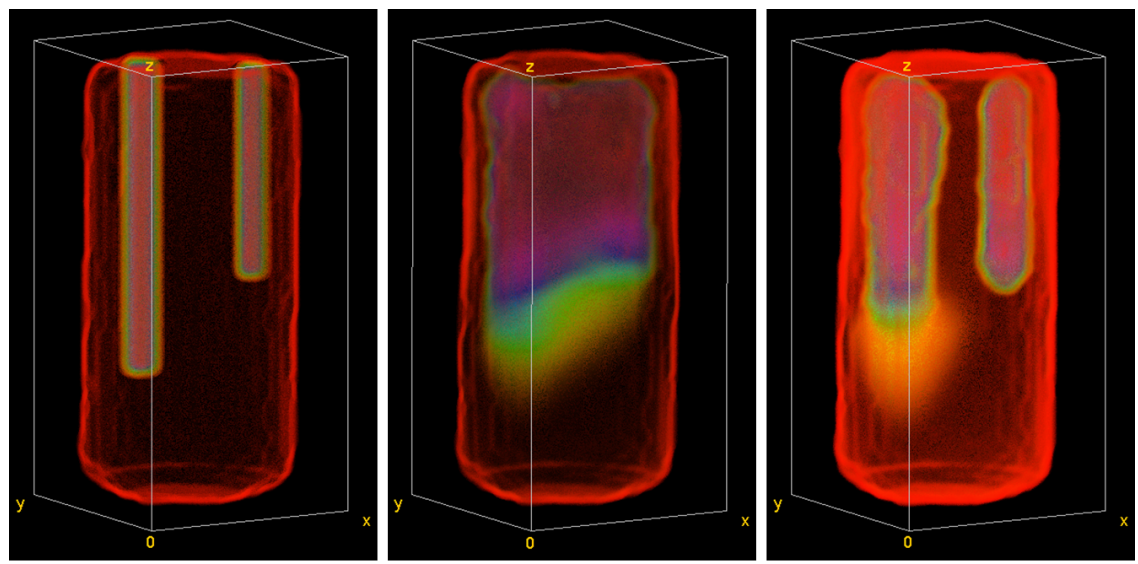

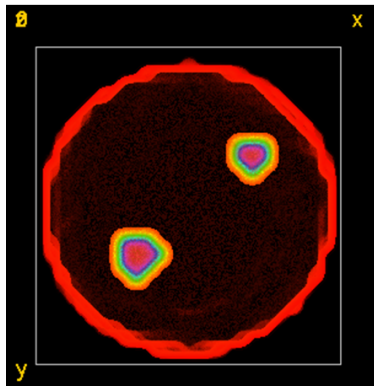

(a)

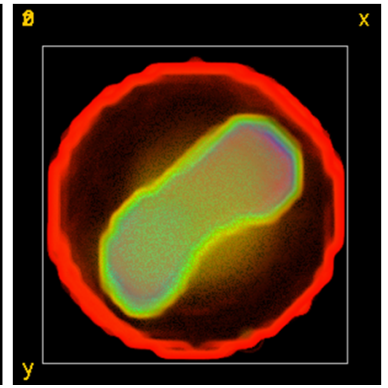

(b)

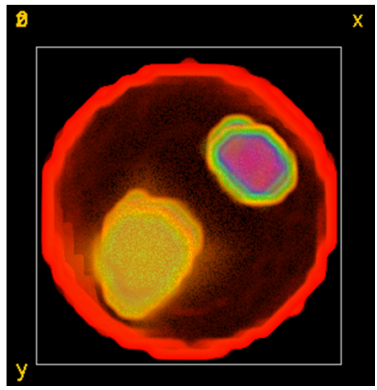

(c)

Figure 4. Fluorescence reconstructions in phantom 1. a) ground truth, b) standard nBorn reconstruction, c) reconstruction obtained with the proposed i-nBorn algorithm. Sparcity priors (14) and (15) were considered.

Table 3. Performance metrics of the fluorescence reconstructions considering sparsity priors, i.e., $\mathcal{R}_{\text {dot }}(\boldsymbol{\eta})=\mathrm{TV}(\boldsymbol{\eta})$ and $\mathcal{R}_{\text {fdot }}(\boldsymbol{\Upsilon})$ given by (15)

\begin{tabular}{ccccc}
\hline Phantom & method & $\rho$ & $\mathcal{C}$ & $\mathcal{Q}$ \\
\hline- & perfect & 1 & $\infty$ & 1 \\
1 & nBorn & 0.305 & 2.28 & $\mathbf{0 . 8 5}$ \\
1 & proposed & $\mathbf{0 . 4 1 6}$ & $\mathbf{3 . 2 5}$ & 0.72 \\
2 & nBorn & 0.197 & 1.96 & 0.17 \\
2 & proposed & $\mathbf{0 . 2 7 4}$ & $\mathbf{2 . 7 9}$ & $\mathbf{0 . 7 9}$ \\
\hline
\end{tabular}

$28 \%$ ) of $\mathcal{C}$ for the sparsity (resp. smooth) priors. Improvements are obtained for both prior types, which suggests the i-nBorn method outperforms the standard nBorn method regardless the penalty functional chosen for regularization. A small decrease of $15 \%$ (resp. $1 \%$ ) of the relative quantification $\mathcal{Q}$ is observed in phantom 1 with sparsity (resp. smooth) priors.

In phantom 2, similar results are obtained. As shown in Fig. 5 and in the bottom row of Fig. 6, the two fluorescent inclusions cannot be separated considering nBornbased reconstruction while they can be well discriminated and localized by means of the proposed method. Specifically, we observe a $39 \%$ increase (resp. $11 \%$ ) of $\rho$ 

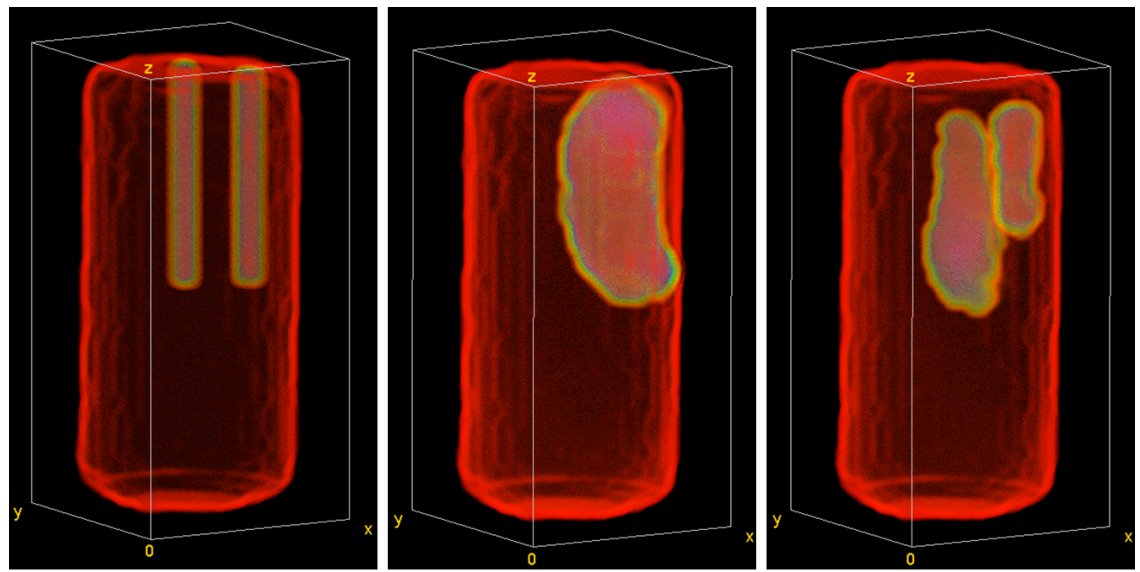

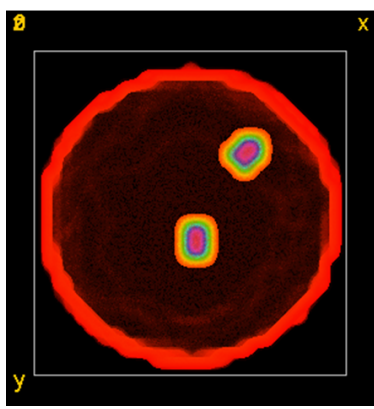

(a)

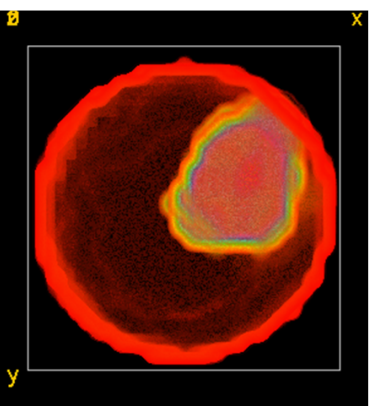

(b)

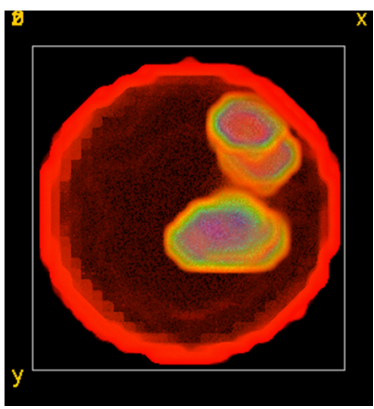

(c)

Figure 5. Fluorescence reconstructions in phantom 2. a) ground truth, b) standard nBorn reconstruction, c) reconstruction obtained with the proposed i-nBorn algorithm. Sparcity priors (14) and (15) were considered.

Table 4. Performance metrics of the fluorescence reconstructions considering regularity priors, i.e., $\mathcal{R}_{\text {dot }}(\boldsymbol{\eta})=\|\Delta \boldsymbol{\eta}\|_{2}^{2}$ and $\mathcal{R}_{\text {fdot }}(\boldsymbol{\Upsilon})=\|\boldsymbol{\Upsilon}\|_{2}^{2}$

\begin{tabular}{ccccc}
\hline Phantom & method & $\rho$ & $\mathcal{C}$ & $\mathcal{Q}$ \\
\hline- & perfect & 1 & $\infty$ & 1 \\
1 & nBorn & 0.343 & 2.58 & $\mathbf{0 . 9 6}$ \\
1 & proposed & $\mathbf{0 . 4 2 4}$ & $\mathbf{3 . 3 1}$ & 0.95 \\
2 & nBorn & 0.211 & 2.11 & 0.37 \\
2 & proposed & $\mathbf{0 . 2 3 5}$ & $\mathbf{2 . 3 6}$ & $\mathbf{0 . 8 7}$ \\
\hline
\end{tabular}

and a $42 \%$ increase (resp. $12 \%$ ) of $\mathcal{C}$ for the sparsity (resp. smooth) priors. The advantage of the proposed method is even more evident due to the higher complexity of the absorbing/scattering heterogeneities distribution. Beyond the higher number of heterogeneities and their variability -both absorbing and scattering inclusions are present, the distance between the two fluorescent inclusions is smaller in phantom 2 than in phantom 1 (see Fig. 1). For this phantom, we observe a dramatic increase of the relative quantification $\mathcal{Q}$, which is increased by a factor of 3.7 for sparsity priors and by a factor of 1.37 for smooth priors.

Finally, we remark that the relative quantification is much better in phantom 1 than 


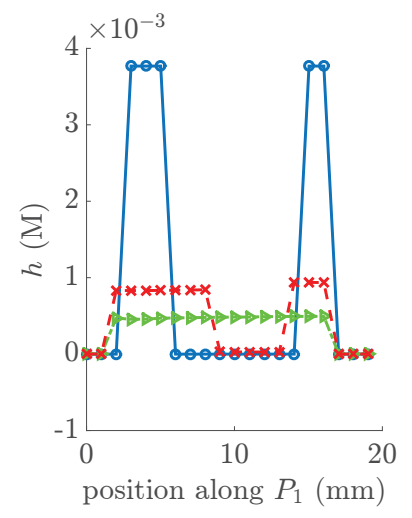

(a)

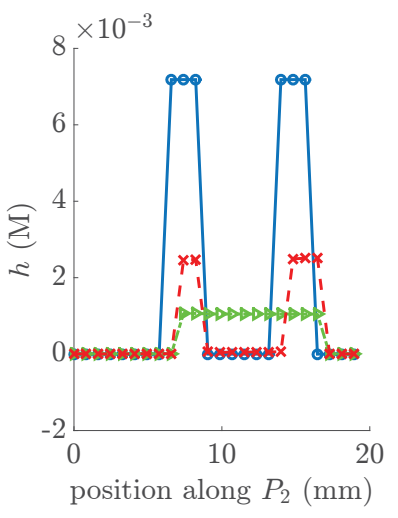

(b)

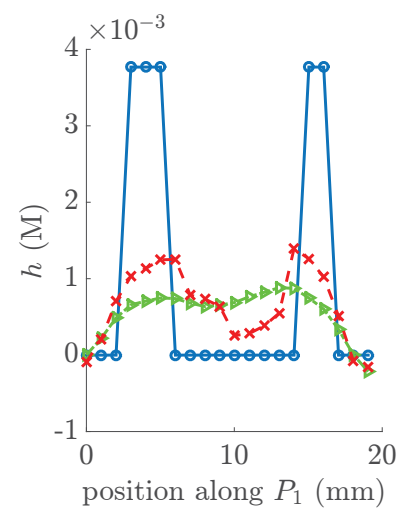

(c)

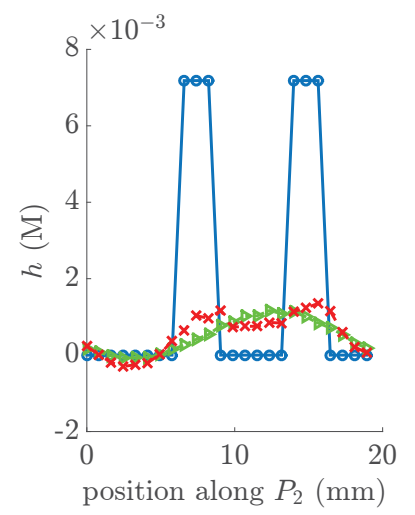

(d)

Figure 6. Reconstructed fluorescence profiles considering the nBorn approach (green line marked with $\triangleright$ ) and our method (red line marked with $\times$ ). The phantom profiles are provided for comparison (blue solid line marked with $\circ$ ). In (a) and (b), the sparsity priors $\mathcal{R}_{\text {dot }}(\boldsymbol{\eta})=\mathrm{TV}(\boldsymbol{\eta})$ and $\mathcal{R}_{\text {fdot }}$ given in (15) are considered. In (c) and (d), the smooth priors $\mathcal{R}_{\text {dot }}(\boldsymbol{\eta})=\|\Delta \boldsymbol{\eta}\|_{2}^{2}$ and $\mathcal{R}_{\text {fdot }}(\boldsymbol{\Upsilon})=\|\boldsymbol{\Upsilon}\|_{2}^{2}$ are chosen. Profiles are plotted along path $\mathcal{P}_{1}$ (see (a) and (c)) and path $\mathcal{P}_{2}$ (see (b) and (d)). For each of the four cases, the reconstructed fluorescence distributions have been normalized to a unit marker quantity such that $\int h(\mathbf{r}) \mathrm{d} \Omega=1 \mu \mathrm{mol}$.

in phantom 2 when the standard nBorn method is considered while the proposed i-nBorn algorithm tends to provide similar quantification capabilities whatever the complexity of the phantom and the priors used in the reconstruction algorithm.

\section{Discussion}

Our approach represents a simple and effective way to experimentally capture the optical inhomogeneities needed to accurately model light propagation within biological tissues. We demonstrated that an optical inhomogeneity map can be recovered from CW measurements in tissue mimicking phantoms. Even without discriminating absorbing from scattering inclusions, the proposed method proved efficient to improve the quality of the fluorescence reconstructions. In particular, an increase of the CNR and correlation of the reconstruction with the ground truth is observed in all our measurements. The dramatic loss of quantification capabilities, which has already been reported elsewhere for the nBorn approach in the presence of complex phantoms [17], can be alleviated considering our approach. This could be a fundamental step towards applications such as follow-up studies in oncology.

It is worth noting that, in this study, only one illumination pattern was considered per view angle, which allowed the acquisition time to be drastically reduced compared to standard illumination schemes based on point source scanning. While the nBorn approach failed to provide satisfactory reconstruction with such an illumination scheme, the i-nBorn provided improved resolution, which could contribute to the emergence of fast acquisition schemes. 
Theses results confirm the importance to incorporate the optical heterogeneities into the forward model involved in FDOT reconstructions [30;31; 37; 38; 62]. They are consistent with those showing that the nBorn method only partially compensate the fluorescence reconstruction errors due to the presence of optical heterogeneities, especially in the presence of scattering inclusions [17; 18]. The advantage of the proposed i-nBorn algorithm is particularly evident in the case of phantom 2 that contains both absorbing and scattering heterogeneities as well as close fluorescent inclusions. It is worth noting that also in the simpler phantom 1, the nBorn method is outperformed by our approach. We anticipate that the advantage of the proposed approach over the standard nBorn method would be even stronger if an anatomical prior obtained by a concurrent anatomical modality is available.

The i-nBorn method can be understood as a generalisation of the standard nBorn method. Indeed, the i-nBorn method is equivalent to the nBorn method for homogeneous samples. The nBorn method provides a preliminary fluorescence reconstruction that can be improved by reconstructing the optical inhomogeneity map of the sample. It is worth reminding that the two advantages offered by the nBorn normalization, namely independence to experimental gains and optical inhomogeneity compensation, are preserved by the i-nBorn method.

As commonly assumed (see for instance [2-8]), the optical properties of the sample were considered to be the same at both excitation and fluorescence wavelengths. For applications where strong variations of optical properties are encountered, the inhomogeneity map can be reconstructed at both wavelengths. However, this comes at the cost of an extra acquisition that consists in illuminating the sample at the fluorescence wavelength. In our phantoms, $\mu_{\mathrm{a}}$ is constant while $\mu_{\mathrm{s}}^{\prime}$ shows as decrease of about $10 \%$ between $633 \mathrm{~nm}$ and $700 \mathrm{~nm}$ [63]. Although the decrease of $\mu_{\mathrm{s}}^{\prime}$ is neglected, our results show that the recovered fluorescence distribution is in very good agreement with the ground truth distribution, which suggests that our method is robust to optical properties variations.

It is important to note that our method is not primarily designed to output an optical map. The inhomogeneity map we recover aims at correcting for the artefacts that occur in the fluorescence images due to inaccuracies of the homogeneous forward model. Demonstrating such a model correction effect is beyond the scope of this paper but it one of the future works that we would like to carry out. Other ideas to correct for inaccuracies are to use approximation error model or marginalize out errors [64;65].

Finally, it is worth mentioning that the possibility to localize optical heterogeneities by means of CW measurements, even without discriminating between absorbing and scattering inclusions, can be of interest for different applications based on optical contrast, e.g. optical oxymetry [66] and mammography [67]. 


\section{Conclusion}

In this paper, we demonstrated the feasibility and advantage of a novel FDOT reconstruction algorithm, which is designed for highly heterogeneous samples. We showed that the inaccuracies of the homogeneous forward model affect the fluorescence images, unless they are corrected for. By reconstructing an optical inhomogeneity map from CW measurements, it is possible to fit the inaccuracies and improve the florescence reconstruction quality. Further studies would help to establish the limits of use of the i-nBorn method and provide in vivo validations. In particular, we believe that the i-nBorn method together with the proposed algorithm can be of particular interest in combination with non-optical techniques, such as MRI and CT. In such a multimodal scheme, the non-optical modality would provide high resolution a priori about the localization of the optical heterogeneities, while DOT measurements could provide an in situ optical characterization of the inhomogeneities. The combination of both types of optical information could be a fundamental step towards improved 3D resolution and quantification of fluorescence markers in vivo.

\section{Acknowledgments}

The research leading to these results has received funding from LASERLAB-EUROPE (grant agreement no. 284464, EC's Seventh Framework Programme). This work was performed within the framework of the LABEX PRIMES (ANR-11-LABX-0063) of Université de Lyon, within the program "Investissements d'Avenir" (ANR-11-IDEX0007) operated by the French National Research Agency (ANR).

\section{Appendix A. Proof of the weight matrix formula}

The pseudo photon density $\psi$ obeys the following coupled equations [40]:

$$
\begin{aligned}
& \mathcal{H} \psi^{\mathrm{x}}=s \text { in } \Omega \\
& \mathcal{H} \psi^{\mathrm{f}}=\psi^{\mathrm{x}} \Upsilon \text { in } \Omega .
\end{aligned}
$$

where $\mathcal{H}$ is the Helmholtz operator with Robin boundary conditions on $\partial \Omega$. The optical properties are assumed to be the same at both excitation and fluorescence wavelengths.

Let $\Sigma$ be the portion of object surface viewed by the camera. Measurements are obtained from the photon density by means of the $\mathbb{R}(\Omega) \rightarrow \mathbb{R}(\Sigma)$ measurement operator $\mathcal{M}$. We have

$$
m=\mathcal{M} \psi
$$

Any compressed measurement $\Gamma^{\mathrm{n}}$ is the projection of the uncompressed measurement $m^{\mathrm{n}}$ onto some detection pattern $d$. We have:

$$
\Gamma^{\mathrm{n}}=\left\langle m^{\mathrm{n}}, d\right\rangle_{\Sigma}
$$


where $\langle\cdot, \cdot\rangle_{\Sigma}$ denotes the $L^{2}$-inner product, i.e. $\langle f, g\rangle_{\Sigma}=\int_{\Sigma} f(\mathbf{x}) g(\mathbf{x}) \mathrm{d} \mathbf{x}$. By definition of $m^{\mathrm{n}}$, we have

$$
m=\left\langle\frac{m^{\mathrm{f}}}{m^{\mathrm{x}}}, d\right\rangle_{\Sigma}
$$

Using (A.2), we have

$$
\begin{aligned}
m & =\left\langle\frac{\mathcal{M} \psi^{\mathrm{f}}}{\mathcal{M} \psi^{\mathrm{x}}}, d\right\rangle_{\Sigma} \\
& =\left\langle\mathcal{M} \psi^{\mathrm{f}}, \frac{d}{\mathcal{M} \psi^{\mathrm{x}}}\right\rangle_{\Sigma} .
\end{aligned}
$$

Introducing the Green's operator of the Helmholtz Eqs. (A.1) leads to

$$
m=\left\langle\mathcal{M G}^{\mathrm{f}}\left[\psi^{\mathrm{x}} \Upsilon\right], \frac{d}{\mathcal{M} \psi^{\mathrm{x}}}\right\rangle_{\Sigma} .
$$

By definition of the adjoint operator $\mathcal{G}^{\mathrm{f} *}$, we have:

$$
=\left\langle\psi^{\mathrm{x}} \Upsilon, \mathcal{G}^{\mathrm{f} *} \mathcal{M}^{*}\left[\frac{d}{\mathcal{M} \psi^{\mathrm{x}}}\right]\right\rangle_{\Omega}
$$

Isolating $\Upsilon$ that is the quantity of interest, we obtain

$$
m=\left\langle\psi^{\mathrm{x}} \mathcal{G}^{\mathrm{f} *} \mathcal{M}^{*}\left[\frac{d}{\mathcal{M} \psi^{\mathrm{x}}}\right], \Upsilon\right\rangle_{\Omega}
$$

The operator $\mathcal{G}^{\text {f }}$ being self adjoint, we obtain

$$
m=\left\langle\psi^{\mathrm{x}} \mathcal{G}^{\mathrm{f}} \mathcal{M}^{*}\left[\frac{d}{\mathcal{M} \psi^{\mathrm{x}}}\right], \Upsilon\right\rangle_{\Omega}
$$

In practice, a measurement may be modelled as

$$
\mathcal{M} \psi=f(\mathbf{r}) \psi(\mathbf{r}), \quad \forall \mathbf{r} \in \Sigma
$$

where $f \in \mathbb{R}(\Sigma)$ depends on the optical properties, the geometry of acquisition and some gain factors. By definition of the adjoint operator that satisfies $\langle\mathcal{M} \psi, m\rangle_{\Sigma}=$ $\left\langle\psi, \mathcal{M}^{*} m\right\rangle_{\Omega}, \forall \psi \in \mathbb{R}(\Omega), m \in \mathbb{R}(\Sigma)$, we have

$$
\mathcal{M}^{*} m= \begin{cases}f(\mathbf{r}) m(\mathbf{r}), & \forall \mathbf{r} \in \Sigma \\ 0 & \forall \mathbf{r} \in \Omega \backslash \Sigma\end{cases}
$$

Inserting (A.11) and (A.12) into (A.10) leads to :

$$
m=\left\langle\psi^{\mathrm{x}} \mathcal{G}^{\mathrm{f}}\left[\frac{\left.d\right|_{\Omega}}{\psi^{\mathrm{x}}}\right], \Upsilon\right\rangle_{\Omega}
$$

where $\left.d\right|_{\Omega}=d$ for $\mathbf{r} \in \Sigma$ and 0 for $\mathbf{r} \in \Omega \backslash \Sigma$. Discretizing the previous equation completes the proof. 


\section{References}

[1] Cherry S R 2004 Physics in Medicine and Biology 49 R13-R48

[2] Ntziachristos V, Bremer C and Weissleder R 2003 European radiology 13 195-208 ISSN 0938-7994 URL http://www.ncbi.nlm.nih.gov/pubmed/12541130

[3] Stuker F, Ripoll J and Rudin M 2011 Pharmaceutics 3 229-274 ISSN 1999-4923 URL http://www. mdpi.com/1999-4923/3/2/229/

[4] Chen J, Venugopal V, Lesage F and Intes X 2010 Opt. Lett. 35 2121-2123 URL http://ol.osa.org/abstract. cfm?URI=ol-35-13-2121

[5] Ducros N, D'andrea C, Valentini G, Rudge T, Arridge S and Bassi A 2010 Opt. Lett. 35 3676-3678 URL http://ol osa.org/abstract.cfm?URI=ol-35-21-3676

[6] Zacharakis G, Ripoll J, Weissleder R and Ntziachristos V 2005 IEEE Transactions on Medical Imaging 24 878-885 URL <GotoISI>://000230218800007

[7] Lasser T, Soubret A, Ripoll J and Ntziachristos V 2008 Medical Imaging, IEEE Transactions on 27 188-194 ISSN 1558-254X

[8] Ducros N, Bassi A, Valentini G, Schweiger M, Arridge S and D'Andrea C 2011 Opt. Lett. 36 1377-1379 URL http://ol.osa.org/abstract.cfm?URI=ol-36-8-1377

[9] Arridge S R and Schotland J C 2009 Inverse Problems 25123010 (59pp) URL http://stacks.iop.org/0266-5611/25/123010

[10] Mozumder M, Tarvainen T, Arridge S, Kaipio J P, d'Andrea C and Kolehmainen V 2015 submitted URL http://arxiv.org/abs/1501.00384

[11] Schweiger M, Arridge S R, Hiraoka M and Delpy D T 1995 Medical Physics 22 1779-1792 URL http://link.aip.org/link/?MPH/22/1779/1

[12] Guven M, Zhou L, Reilly-Raska L and Yazici B 2010 Medical Imaging, IEEE Transactions on $29230-245$ ISSN 0278-0062

[13] Grzywacz T, Sikora J and Wojtowicz S 2008 Magnetics, IEEE Transactions on 44 1374-1377 ISSN 0018-9464

[14] Elisee J, Gibson A and Arridge S 2010 Biomedical Engineering, IEEE Transactions on 57 2737-2745 ISSN 0018-9294

[15] Ntziachristos V and Weissleder R 2001 Opt. Lett. 26 893-895 URL http://ol. osa. org/abstract. cfm?URI=ol-26-12-893

[16] Soubret A, Ripoll J and Ntziachristos V 2005 Ieee Transactions on Medical Imaging 24 1377-1386 URL <GotoISI>://000232236800013

[17] Pyka T, Schulz R, Ale A and Ntziachristos V 2011 Opt. Lett. 36 4329-4331 URL http://ol.osa.org/abstract. cfm?URI=ol-36-22-4329

[18] Abascal J F P J, Aguirre J, Chamorro-Servent J, Schweiger M, Arridge S, Ripoll J, Vaquero J J and Desco M 2012 Journal of Biomedical Optics 17 036013-1-036013-9 URL +http://dx.doi.org/10.1117/1.JBO.17.3.036013 
[19] Schulz R, Ale A, Sarantopoulos A, Freyer M, Soehngen E, Zientkowska M and Ntziachristos V 2010 IEEE Transactions on Medical Imaging 29 465-473 ISSN 0278-0062

[20] Guo X, Liu X, Wang A, Tian F, Liu F, Zhang B, Hu G and Bai J 2010 Biomedical Engineering, IEEE Transactions on 57 2876-2883 ISSN 0018-9294

[21] Liu X, Guo X, Liu F, Zhang Y, Zhang H, Hu G and Bai J 2011 Biomedical Engineering, IEEE Transactions on 58 2139-2143 ISSN 0018-9294

[22] Mohajerani P, Hipp A, Willner M, Marschner M, Trajkovic-Arsic M, Ma X, Burton N, Klemm U, Radrich K, Ermolayev V, Tzoumas S, Siveke J, Bech M, Pfeiffer F and Ntziachristos V 2014 Medical Imaging, IEEE Transactions on 33 1434-1446 ISSN 0278-0062

[23] Zhang X, Toronov V Y and Webb A G 2006 Review of Scientific Instruments 77 114301-114301-8 ISSN 0034-6748

[24] Stuker F, Baltes C, Dikaiou K, Vats D, Carrara L, Charbon E, Ripoll J and Rudin M 2011 IEEE Transactions on Medical Imaging 30 1265-1273

[25] Solomon M, Nothdruft R E, Akers W, Edwards W B, Liang K, Xu B, Suddlow G P, Deghani H, Tai Y C, Eggebrecht A T, Achilefu S and Culver J P 2013 Journal of nuclear medicine : official publication, Society of Nuclear Medicine 54 639-46 ISSN 1535-5667 URL http://www.ncbi.nlm.nih.gov/pubmed/23447655

[26] Zhang B, Liu S, Cao X, Liu F, Wang X, Luo J, Shan B and Bai J 2013 Multimedia, IEEE Transactions on 15 1031-1038 ISSN 1520-9210

[27] Baritaux J C, Hassler K and Unser M 2010 Medical Imaging, IEEE Transactions on 291075 -1087 ISSN 0278-0062

[28] Baritaux J C, Hassler K, Bucher M, Sanyal S and Unser M 2011 Medical Imaging, IEEE Transactions on 301143 -1153 ISSN 0278-0062

[29] Holt R W, Davis S and Pogue B W 2013 Opt. Lett. 38 2407-2409 URL http: //ol.osa.org/abstract. cfm?URI=01-38-14-2407

[30] Tan Y, Cao Zand Sajja H K, Lipowska Mand Wang Y A Y L and Jiang H 2013 Journal of X-Ray Science and Technology 214352 URL http://doi.org/10. 3233/XST-130365

[31] Zhang J, Chen D, Liang J, Xue H, Lei J, Wang Q, Chen D, Meng M, Jin Z and Tian J 2014 Biomed. Opt. Express 5 1861-1876 URL http://www . osapublishing.org/ boe/abstract. cfm?URI=boe-5-6-1861

[32] Hielscher A, Klose A and Hanson K 1999 Medical Imaging, IEEE Transactions on 18 262-271 ISSN 0278-0062

[33] Ntziachristos V, Hielscher A H, Yodh A G and Chance B 2001 Ieee Transactions on Medical Imaging 20 470-478 URL <GotoISI> ://000169452900002

[34] Tan Y and Jiang H 2008 Appl. Opt. 47 2011-2016 URL http://ao.osa.org/ abstract. cfm?URI=ao-47-12-2011 
[35] Prakash J, Dehghani H, Pogue B and Yalavarthy P 2014 Medical Imaging, IEEE Transactions on 33 891-901 ISSN 0278-0062

[36] Prakash J, Shaw C, Manjappa R, Kanhirodan R and Yalavarthy P 2014 Selected Topics in Quantum Electronics, IEEE Journal of 20 74-82 ISSN 1077-260X

[37] Wu L, Zhao H, Wang X, Yi X, Chen W and Gao F 2014 Appl. Opt. 53 6970-6982 URL http://ao.osa.org/abstract.cfm?URI=ao-53-30-6970

[38] Gremse F, Theek B, Kunjachan S, Lederle W, Pardo A, Barth S, Lammers T, Naumann U and Kiessling F 2014 Theranostics 4 960-971 URL http://dx.doi. org/10.7150/thno. 9293

[39] Arridge S R and Lionheart W R B 1998 Opt. Lett. 23 882-884 URL http: //ol .osa.org/abstract. cfm?URI=ol-23-11-882

[40] Correia T, Ducros N, D'Andrea C, Schweiger M and Arridge S 2013 Opt. Lett. 38 1903-1905 URL http://ol.osa.org/abstract.cfm?URI=ol-38-11-1903

[41] D'Andrea C, Ducros N, Bassi A, Arridge S and Valentini G 2010 Biomed. Opt. Express 1 471-481 URL http://www.opticsinfobase.org/boe/abstract.cfm? URI=boe-1-2-471

[42] Ducros N, Bassi A, Valentini G, Canti G, Arridge S and D'Andrea C 2013 Journal of Biomedical Optics 18 020503-020503 URL +http://dx.doi.org/10.1117/1. JBO . 18.2.020503

[43] Wang D, Liu X, Liu F and Bai J 2010 Information Technology in Biomedicine, IEEE Transactions on 14 1346-1354 ISSN 1089-7771

[44] Mohajerani P and Ntziachristos V 2013 Opt. Lett. 38 2324-2326

[45] Jin A, Yazici B and Ntziachristos V 2014 Image Processing, IEEE Transactions on 23 2609-2624 ISSN 1057-7149

[46] Konecky S D, Panasyuk G Y, Lee K, Markel V, Yodh A G and Schotland J C 2008 Opt. Express 16 5048-5060 URL http://www.opticsexpress.org/abstract. cfm?URI=oe-16-7-5048

[47] Zacharopoulos A D, Svenmarker P, Axelsson J, Schweiger M, Arridge S R and Andersson-Engels S 2009 Opt. Express 17 3042-3051 URL http://www. opticsexpress.org/abstract. cfm?URI=oe-17-5-3042

[48] Rudge T J, Soloviev V Y and Arridge S R 2010 Opt. Lett. 35 763-765 URL http: //ol .osa.org/abstract.cfm?URI=ol-35-5-763

[49] Hyde D, Miller E, Brooks D and Ntziachristos V 2010 Medical Imaging, IEEE Transactions on 29365 -374 ISSN 0278-0062

[50] Cao X, Zhang B, Liu F, Wang X and Bai J 2011 Opt. Lett. 36 4515-4517 URL http: //ol.osa.org/abstract. cfm?URI=ol-36-23-4515

[51] Shaw C B and Yalavarthy P K 2012 Journal of Biomedical Optics 17086009 086009 URL http://dx.doi.org/10.1117/1.JBO.17.8.086009 
[52] Lee O K, Kim J M, Bresler Y and Ye J C 2011 Medical Imaging, IEEE Transactions on 30 1129-1142 ISSN 0278-0062

[53] Kim D, Sra S and Dhillon I 2010 SIAM Journal on Scientific Computing 32 3548-3563 (Preprint http://epubs.siam.org/doi/pdf/10.1137/08073812X) URL http://epubs.siam.org/doi/abs/10.1137/08073812X

[54] Correia T, Aguirre J, Sisniega A, Chamorro-Servent J, Abascal J, Vaquero J J, Desco M, Kolehmainen V and Arridge S 2011 Biomed. Opt. Express 2 2632-2648 URL http: //www .opticsinfobase.org/boe/abstract.cfm?URI=boe-2-9-2632

[55] Black M, Sapiro G, Marimont D and Heeger D 1998 Image Processing, IEEE Transactions on 7 421-432 ISSN 1057-7149

[56] Douiri A, Schweiger M, Riley J and Arridge S R 2007 Measurement Science and Technology 1887 URL http://stacks.iop.org/0957-0233/18/i=1/a=011

[57] Schweiger M and Arridge S 2014 Journal of biomedical optics 19040801 ISSN 1560-2281 URL http://www.ncbi.nlm.nih.gov/pubmed/24781586

[58] Ducros N, D'Andrea C, Bassi A, Valentini G and Arridge S 2012 Physics in Medicine and Biology 573811 URL http://stacks.iop.org/0031-9155/57/i= $12 / a=3811$

[59] Bassi A, Farina A, D'Andrea C, Pifferi A, Valentini G and R C 2007 Opt. Express, 151448214487.

[60] Fang Q and Boas D 2009 Tetrahedral mesh generation from volumetric binary and grayscale images Biomedical Imaging: From Nano to Macro, 2009. ISBI '09. IEEE International Symposium on pp 1142-1145 ISSN 1945-7928

[61] http://statweb.stanford.edu/ wavelab/ URL http://statweb.stanford.edu/ $\sim$ wavelab/

[62] Koenig A, Planat-Chrtien A, Hassler K, Bucher M, Coutard J G, Herv; L, Brambilla M, Josserand V, Coll J L, Vats D, Dikaiou K, Rudin M and Dinten J M 2012 Validation of an xct/fdot system on mice URL http://dx.doi.org/10.5402/ $2012 / 735231$

[63] Pifferi A, Torricelli A, Cubeddu R, Quarto G, Re R, Sekar S K V, Spinelli L, Farina A, Martelli F and Wabnitz H 2015 Journal of Biomedical Optics 20121304 URL http://dx.doi.org/10.1117/1.JB0.20.12.121304

[64] Tarvainen T, Kolehmainen V, Kaipio J P and Arridge S R 2010 Biomed. Opt. Express 1 209-222 URL http://www.opticsinfobase.org/boe/abstract.cfm? URI=boe $-1-1-209$

[65] Kolehmainen V, Tarvainen T, Arridge S R and Kaipio J P 2010 International Journal for Uncertainty Quantification 1117

[66] Ferrari M and Quaresima V 2012 NeuroImage 63 921-35 ISSN 1095-9572 URL http://www.ncbi.nlm.nih.gov/pubmed/22510258

[67] Pifferi A, Farina A, Torricelli A, Quarto G, Cubeddu R and Taroni P 2012 J. Near Infrared Spectrosc. 20 